\title{
Internet of Things-based innovations in Saudi healthcare sector: A methodological approach for investigating adoption issues
}

\author{
Feisal Hadi Masmali \\ Newcastle Business School, The \\ University of Newcastle, New South \\ Wales, Australia \\ College of Business Administartion, \\ Jazan University, Saudi Arabia \\ fmasmali@jazanu.edu.sa
}

\author{
Shah Jahan Miah \\ Newcastle Business School, \\ The University of Newcastle, \\ Newcastle City Campus, \\ New South Wales, Australia \\ shah.miah@newcastle.edu.au
}

\author{
Nada Yahya Mathkoor \\ Engineering School, The \\ University of Melbourne, \\ Melbourne, Australia \\ nmathkoor@student.unimelb \\ .edu.au
}

\begin{abstract}
The Internet of Things (IoT) has proliferated over the last few years as the next-generation technologies that impact both human systems and businesses. Using today's Internet network capacities, this technology has extended various benefits in healthcare sectors. For instance, existing studies already indicated that information technology (IT) applications with IoTbased innovations may revolutionize the healthcare industry and subsequently help to improve the real-time reporting of patients' health data. It should be noted that the adoption of IoT and its relevant interventions in the health sector has not been as fast as the uptake been observed in other industries. To tackle this issue, we develop a qualitative phenomenological approach for investigating factors that affect IoT adoption and its integration into healthcare service delivery in Saudi Arabia. To this end, the study aims to reveal the practical experiences of healthcare professionals or service administrators in using IoT devices as they deliver medical services.
\end{abstract}

Keywords - IoT, Diffusion of Innovation, Healthcare services, Technology acceptance model, Structural Equation Model, Saudi Arabia, Healthcare innovation

\section{INTRODUCTION}

Internet of Things (IoT) is a term that Kevin Ashton coined in 1999 to describe a system in which the "internet is used to empower computers to empower the world for themselves" [1]. This view is associated to machines, infrastructure and humans through cloud computing [1]. Researchers such as, Breivold and Sandström [2] stated in their research that IoT helps connect every day apparatuses and devices that we use at home as well as industrial equipment. As many organizations as well as governments have realised the value of using IoT in their practices, the present study is intended to investigating factors that affect IoT adoption and its integration into healthcare service delivery in Saudi Arabia.

IoT research have significant association to e-government studies. E-government literature has grown rapidly over the past few decades in publication administration [3-5], agricultural service $[6,7]$, transportation [8, 9] and healthcare service [10-12]. However, e-government application in the healthcare sector have been limited and especially so for developing nations, due to many issues such as lack of investment and proper awareness of using the latest technologies for improving people's performance. This research makes an important contribution to the current egovernment literature by creating a new direction of using IoT for enhancing e-government services specifically for healthcare, which involves adopting other latest mobile technologies. The system responds to the demand to understand the contexts in which egovernment is implemented and especially with reference to IoT [13]; Shareef et al., 2016; Venkatesh et al., 2016; Wirtz \& Daiser, 2018). The system will be very useful for establishing a better comprehension of using IoT in healthcare systems in other developing countries.

This paper extends TAM so that the adoption of IoT is better understood by examining the role of contextual factors in improving IoT in healthcare services. Current TAM-based IoT models concentrate mainly on human variables and devices' characteristics. This research illustrates the applicability of the expanded system to investigate IoT in the healthcare sector with empirical evidence in the developing countries' context. This study offers important research results for e-government improvements in Saudi Arabia. The significance of this study for e-government developers lies in providing them with valuable knowledge so that e-government healthcare strategies are effective and practical. This study will be conducted within the domain of Saudi's public healthcare sector. Under this public health care sector there are three different areas that we identified. One area is government public health which is the main channel of service delivery for the benefit of the rural and urban citizens. It is related to provide healthcare services ensuring community health support, advice and improvement for citizens.

\section{THEORETICAL PERSPECTIVES}

A theory is a collection of associations referring to a specific phenomenon, explained by a system of actions, events, processes and ideas [14]. The objective of a theoretical analysis is to deliver clear views on the causal relationships between certain constructs [7]. The key purpose of using a theory in the analysis is to: (a) describe a certain phenomenon, its causal relationships and its boundaries; (b) clarify how, why and when certain events are occurring; (c) provide approximate forecasts of what will occur under certain conditions; and (d) provide detailed guidance for constructing a certain phenomenon. A conceptual structure to address a particular problem typically needs to be built to help describe a theory [15]. This research seeks to establish a methodological structure focused on a systematic analysis of IoTrelated literature and its implementation from a range of perspectives, with specific reference to Saudi Arabia's healthcare services. It promotes the qualitative and quantitative approaches to achieve the study objectives. These are explained in more detail below. 
This study's qualitative approach is established in the diffusion of innovation (DOI) concept, which assumes the propagation of new notions (innovations) among those who are within social system is through certain channels as time passes [16]. This study's interview questions were crafted using these factors while the analysis of the interview data was towards ascertaining the most remarkable factor(s) affecting innovation diffusion. The conceptual framework is made up of the beliefs, theories, assumptions as well as anticipations that that supports and informs this study.

Referring to the quantitative method, the goal of this study is to examine the critical factors for IoT adoption in Saudi Arabia from the citizens' perspectives. A conceptual framework TAM - is devised to achieve the objectives of this analysis. TAM has been chosen for a number of reasons. First, it may provide valuable insights into technology adoption for people using egovernment services directly [12]. Second, TAM can be applied flexibly to the IoT adoption in healthcare service studies [12]; [17]; [18]. Third, TAM is robust and accurate in predicting e-government adoption [19]; [20]; [21]. Fourth, TAM confirms that great strides are being made in understanding the use of unique innovations like IoT [22].

\section{BASIS OF THEORETICAL FRAMEWORKS}

This research used DOI theory to explore healthcare administrators' diffusion and adoption of the IoT for delivering healthcare services. DOI theory comprises four key elements, namely, innovation, communication channels, time and social systems [16]. Innovation pertains to the notion, practice or object that a person or other units of adoption see as new [16]. The theory also underscores the importance of perception given that the manner in which users or social systems perceive a given idea determines whether such a notion will be regarded as a noble or desired concept [16]. Thus, it is possible for an idea to have existed for many years and still be viewed as new by people or social systems.

\section{DIFFUSION OF INNOVATION THEORY}

According to the postulation of the DOI theory, the diffusion process has to do with the movement of new notions (innovations) via some vehicles among the people in a social system [23]. A critical part of this procedure is innovation that comes with the execution of new notions characterised by creating organisational value [24]. New notions can exist as services, systems or procedures or even the improvement of the existing ones. The manner in which new notions (innovations) spread (diffusion) is unique. Researchers need to focus such uniqueness towards the identification of the reasons for quicker proliferations of innovations relative to others.

Compatibility, relative advantage, trialability, complexity and observability directly influence the acceptance of innovations by individuals; that is, an innovation is regarded as superior to a mere idea. Relative advantage is measured on the basis of the social prestige, convenience and satisfaction derived from an innovation, apart from the economic advantages offered by it. Compatibility refers to the status of consistency with existing values and previous experiences and requirements of possible adopters, while complexity has to do with the extent to which an innovation is perceived as easy to comprehend and utilise. Trialability refers to the degree to which an innovation can be subjected to experimentation on a limited basis, and observability is the extent of the visibility of an innovation's outcomes to others.

Studies related to organisational analysis often apply DOI theory in describing the basic patterns in adopting IT innovations [25]; [26]. Rogers [16] who introduced DOI theory looked into innovation adoption as the decision of taking and using new creations. His observation shows that innovation diffusion is strongly dependent on human capital and that there is almost a normal distribution of the number of people who adopt an invention with time.

Wamba [27] carried out a review that suggests DOI theory as a well-known approach in studying IoT adoption. Although it is used extensively in organisational analysis, there have been criticisms regarding this viewpoint due to its individualistic approach in describing organisational behaviours, its inattention to the impacts of environmental factors and its inability to consider aggregate IT implementation [28], [29]. However, some researchers are still supporting this theory. For example the theory was applied in the study of Wamba and Chatfield [30] towards the investigation of the development of value from RFID supply chain projects in logistics and manufacturing domains. Similarly, Quetti, et al. [31] used the theory to examine the RFID adoption procedure in a vertical supply chain with silk industry in Italy being the case study. Liu, et al. [32] also used the theory in the development of a research model intended towards identifying crucial determining factors for the purpose of adopting RFID to deliver public services.

The French sociologist Gabriel Tarde was the first to carry out a study on the diffusion of innovation in 1903 [33]. His research involved the plotting of an S-shaped diffusion curve, which continues to be used today given that the adoption of the majority of new ideas exhibit an S-shaped pattern [33]. Depending on the speed of adoption, the curve's slope can be steep (fast adoption) or gradually inclined (slow adoption) [16]. The S-curve formulated by Tarde was validated by sociologists Bryce Ryan and Neal Gross [34] in their scrutiny of the diffusion of hybrid corn seeds among Iowa farmers in the United States. The cumulative plotting that the authors performed across time demonstrated that the rate at which the hybrid seeds are adopted follows a typical Sshaped curve. Their study motivated further research on the diffusion of innovation (Ryan \& Gross, 1943People belonging to the late majority see innovation adoption as an economic necessity and embrace such technologies mostly because of peer pressure [16]. In this group are individuals who exercise caution during the adoption of a new idea [16]. In the laggard group, people do not forgo conventional practices and procedures, and they are unwilling to adopt novel conceptions [16].

The decision to adopt and use an innovation is preceded by the stages of awareness, interest, evaluation and trial [23]. During the awareness stage, people or social systems become conscious of an innovation but lack information or knowledge concerning it. The interest stage is typified by attentiveness, but this phase is still wanting in any active endeavour to search for further information about the innovation. The evaluation stage involves a mental or systemic assessment of the innovation in order to pinpoint how the invention will benefit its current or future users and deciding on whether to embrace it or not. The trial phase is where the innovation is used in its entirety. Adopters make the decision to use an innovation continually [23], but an important barrier to such espousal is uncertainty, which is brought about by the effects of innovations [35].

There is sufficient corroboration that innovations such as the IoT can help improve the healthcare sector [36]. However, the successful implementation of innovations in a particular region does not necessarily mean that such advances will proliferate rapidly to other locations, if at all. Berwick [36] identified three factors that slow down innovation diffusion in healthcare: perceptions regarding innovations, the characteristics of likely adopters and organisational, managerial and contextual factors [36].

Sauer and Lau [37] declared that adopting any process or product engendered through an information system (IS) necessitates decision-making. Decision-makers are in charge of the adoption of new IS innovations because they have the resources and power/authority to drive people to implement behavioural changes in an organisation. In the healthcare industry, the chief decisionmakers are healthcare administrators who are also responsible for the adoption of IoT in healthcare services. This reality underscores the need to cast light on the lived experiences of such personnel regarding how they use IoT technologies as part of their service provision responsibilities to patients. DOI theory is generally believed to be a valid model of innovation diffusion, especially in the healthcare domain.

\section{PROPOSED CONCEPTUAL FRAMEWORK}

Given these issues, the present work carried out a qualitative phenomenological analysis to fully elucidate the factors that characterise the slow adoption of the IoT by healthcare 
organisations in Saudi Arabia. Specifically, the lived experiences of healthcare providers in their use of IoT technologies as they provide healthcare services is explored in detail [38]. The study site is Jazan, which is located in the south-western region of Saudi Arabia, and the study population consists of administrative personnel who make decisions regarding technology adoption. As stated earlier, the theoretical framework that guided the research was DOI theory. The conceptual framework and its constituent elements are illustrated in Fig. 1.

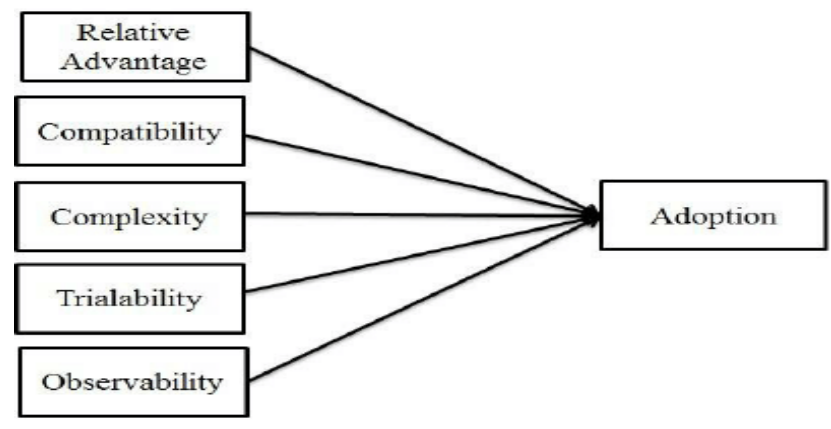

Figure 1: Diffusion of innovation theory (Rogers, 1983)

For the quantitative approach, TAM is broadly acknowledged as a great theory for forecasting the technologies' adoption from an individual viewpoint [39]. It is widely used to explicate why people choose to use a particular technology or not. Two main constructs, namely perceived user-friendliness (PEOU) and perceived userfriendliness (PU) are proposed as the determinants influencing individuals' decision to adopt a technology [40]. PEOU refers to the degree to which a person thinks the use of a technology is easy and requires little effort [40]. PU is related to the degree to which a person believes he can improve his or her performance at work using a technology [40]. Overall, TAM focuses on the beliefs of individuals about adopting a technology [40]. The conceptual structure for this study is shown in Figure 3.1 below, as it is an integral part of discussing the crucial factors for IoT adoption in Saudi Arabia. This research covers four types of TAM extension variables. The four categories suggested concentrate on entity, programme, architecture and context aspects. Individual characteristics include computer productivity, personal creativity and computer anxiety. The system features include quality of services and quality of knowledge. The interface features include PEOU and PU.

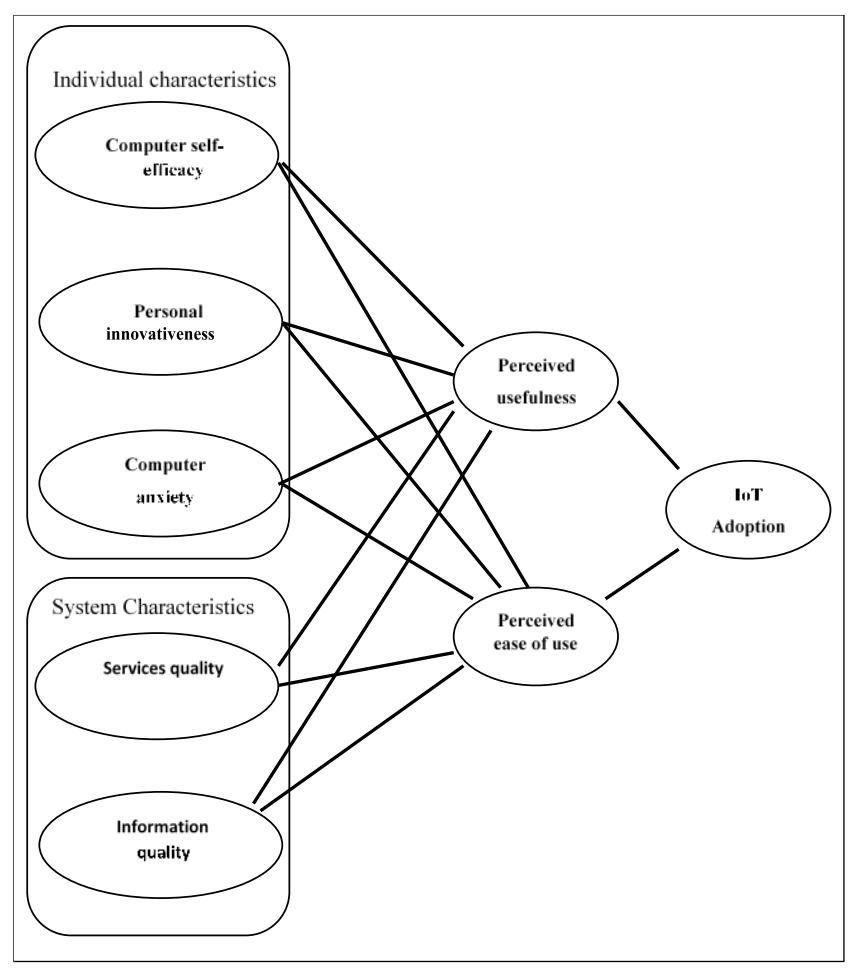

Figure 3: A Conceptual Framework for the Adoption of IoT

VI DATA COLLECTION AND ANALYSIS

This study is planned to collect data using mixed methods, with implementation via semi-structured interviews and survey data. There was the combination of inquiries and procedures while data were obtained from the participants. The analysis of the results was inductive, moving from particular to general issues, and the interpretation of the collected data. In the first phase, the choice of a qualitative method was due to the necessity of exploring a particular phenomenon based on the views and experiences of people.

This research aims to develop an IoT integration framework for healthcare services in Saudi Arabi's e-government development sector. A mixed methodology is used to achieve these research goals. A conceptual framework is developed by assuming the critical factors for effective compliance of IoT integration with healthcare service. The conceptual framework will be tested and validated using structural equation modelling with the use of survey data collected from healthcare administrators in Saudi Arabia. For further validation of the identified critical factors, thematic analysis is conducted on the simultaneously collected semi-structured interview data. The quantitative findings and qualitative findings are triangulated to better understand compliance with healthcare in e-government development of IoT integration in the Kingdom.

Interview is among the most critical data source and proof with the aim of confirming people's perspectives, notions and observations. Many consider an interview as the most remarkable tool to gather deep information about social actors' attitudes, behaviours, observations, knowledge and notions in modern situations [41]. There are three categories under an interview; structured, semi-structured and unstructured designs [42]; [41]. The semi-structured is considered as the most useful and effective in collecting qualitative perceptions, examining and understanding human behaviours [43]; [44]; [41]. The general means of accomplishing this is through open-ended questions, as it permits participants' deep discussion of their experiences and behaviours [43]; [44]. It also offers the chances of understanding a context for exploration and correspondingly connects social situations and social actors' attitudes [42]. To carry out interviews, a critical criterion is participants' agreement to reveal appropriate information regarding their experiences [42]. It is also possible to explore more themes as well as appropriate information [45]. 
Questionnaires, which require participants to respond to an identical set of questions, are important data collection tools [41] as they enable a researcher to identify the variability of a given phenomenon. Although it is possible to use questionnaires as the only data collection instrument in a study, combining it with other methods is recommended under a mixed methods design [42]. Questionnaires are one of the most popular data collection instruments in research on business and education. Many people have experience using questionnaires [41]. For the current study, the questionnaire method was regarded as the best way to acquire relevant quantitative data in the second phase of the investigation.

For this paper, the use of a quantitative study approach is suitable for two purposes. First, by collecting and analysing numerical data, a quantitative methodology may explain the fundamental link between the research constructions [46]; [47]. Secondly, the implementation of such a technique enables the generalisation of study results for large populations [48]; [49]. This study adopts the survey approach using the quantitative data collection technique. It is widely used to investigate the cause of the phenomenon and collect data on sample population attitudes and behaviour [50]; [49]. This research adopts a survey on the basis of its capacity to study (a) the existing trends of IoT adoption in the Kingdom and (b) testing and validating special hypotheses for the framework suggested in this study.

All the data collected in the qualitative stage were examined using thematic content analysis with the help of NVivo software. The data analysis included the examination, organisation, categorisation and interpretation of the data derived from qualitative and quantitative sources [51]. No standardised method or technique has been developed for the analysis of qualitative data [52]. Hence, the most useful analytical approach is selected to achieve the objectives of a study. The term 'qualitative' is often used as a synonym for 'interview'. It refers to the use of nonnumerical data, thus pertaining typically to informational forms other than words, such as pictures or video clips [52]. The basic purposes of qualitative research are the examination of a research topic in accordance with the views and opinions of participants to understand why and how people develop and maintain certain points of view about a situation or problem [53].

During the COVID 19, ensuring that the study does not violate any social isolation and community lockdown for maintaining human spacing, communication and interactions, we redefine new context for conducting the study and set up some variables if they are applicable. We consider the situation of COVID 19 and will make sure that the research will be sensitive to avoid direct human contact. Also, the research will ensure that the appropriate social isolation and the community lockdown process does not violate Saudi and Australia instructions. Additionally, the research will be sensitively maintained. In this case, the ethics application might be considered to be changed.

\section{DISCUSSION AND CONCLUSION}

The proposed methodological approach was designed to capture both the issues that emerged as important themes in the literature review as well as the results of the year one thesis. Responses from the health administrator to the questions illustrate areas that need more study. In other words, utilising the methodological lens described in this paper, the outcomes would give an adequate insights in terms of answering the research questions. Given that the first year results provided potential interviewees for this study, they may be asked to extend and further interpret of the initial responses. The proposed methodology would assist the Saudi government to develop strategies for developing further on research investigation for useful insights generation leading to implementing IoT in healthcare based on the comments elicited by participants in the target community.
The overarching research question is for investigation through the proposed research approach is what are the factors that influence the effective integration of IoT innovations and healthcare service delivery in Saudi Arabia? This investigation is expected to add new and important information about the integration of IoT innovations and healthcare service delivery in Saudi Arabia. IoT is now considered to be advantageous in various industries including agriculture, hospitality, advertising, construction, manufacturing, services and healthcare. Integrating IoT with the existing technical system would revolutionise Saudi Arabia's healthcare/medical system. Since the Saudi government expects to provide the best possible healthcare to its citizens, IoT integration is essential. Integration of IoT in the technical aspects of healthcare will widen the scope of treatment, link devices with people, applications and machines, and help provide better care for patients, make processes more efficient, reduce errors and save much time and avoid delays. The aim of this research is to give a methodological overview to develop a real problem-based scenario for developing an innovative healthcare information system (for example [10], and [11], in future. This research provides an opportunity for future research to develop a new design framework (e.g. [54] and [55]) that influence the adoption of IoT in any form of new healthcare information system design for instance [56] or [57].

\section{REFERENCES}

[1] T. Cole. "Interview with Kevin Ashton-inventor of IoT: Is driven by the users." https://www.smart-industry.net/interview-with-iotinventor-kevin-ashton-iot-is-driven-by-the-users/ (accessed 30).

[2] H. P. Breivold and K. Sandström, "Internet of things for industrial automation--challenges and technical solutions," in 2015 IEEE International Conference on Data Science and Data Intensive Systems, 2015: IEEE, pp. 532-539.

[3] L. C. Serra, L. P. Carvalho, L. P. Ferreira, J. B. S. Vaz, and A. P. Freire, "Accessibility Evaluation of E-Government Mobile Applications in Brazil," (in English), Proceedings of the 6th International Conference on Software Development and Technologies for Enhancing Accessibility and Fighting InfoExclusion, vol. 67, pp. 348-357, 2015, doi: 10.1016/j.procs.2015.09.279.

[4] M. Kurfalı, A. Arifoğlu, G. Tokdemir, and Y. Paçin, "Adoption of egovernment services in Turkey," Computers in Human Behavior, vol. 66, pp. 168-178, 2017.

[5] N. P. Rana, Y. K. Dwivedi, S. Luthra, B. Lal, and M. A. A. Alryalat, "Advances in Electronic Government (e-Government) Adoption Research in SAARC Countries," in Conference on e-Business, $e$ Services and e-Society, 2017: Springer, pp. 147-158.

[6] P. Brous and M. Janssen, "Advancing e-Government using the internet of things: a systematic review of benefits," in International Conference on Electronic Government, 2015: Springer, pp. 156-169.

[7] K. Karunasena and H. Deng, "A Citizen-Oriented Approach for Evaluating the Performance of e-Government in Sri Lanka," (in English), International Journal of Electronic Government Research, vol. 8, no. 1, pp. 43-63, Jan-Mar 2012, doi: 10.4018/jegr.2012010103.

[8] A. Almukhlifi, H. Deng, and B. Kam, "Critical factors for the adoption of e-government in developing countries: validation of a measurement model," in Proceedings of the 12th International Conference on Theory and Practice of Electronic Governance, 2019, pp. 397-407.

[9] X. Gao and J. Lee, "E-Government Services and Social Media Adoption: Experience of Small Local Governments in Nebraska," in Proceedings of the 18th Annual International Conference on Digital Government Research, 2017: ACM, pp. 584-585.

[10] F. H. Masmali and S. J. Miah, "Adoption of IoT based innovations for healthcare service delivery in Saudi Arabia," 2019.

[11] S. J. Miah, "A new semantic knowledge sharing approach for egovernment systems," in 4th IEEE International Conference on Digital Ecosystems and Technologies, 2010: IEEE, pp. 457-462.

[12] S. H. P. Shyu and J. H. Huang, "Elucidating usage of e-government learning: A perspective of the extended technology acceptance model," (in English), Government Information Quarterly, vol. 28, no. 4, pp. 491-502, Oct 2011, doi: 10.1016/j.giq.2011.04.002.

[13] Y. K. Dwivedi, N. P. Rana, H. Chen, and M. D. Williams, "A Metaanalysis of the Unified Theory of Acceptance and Use of Technology (UTAUT)," in IFIP international working conference on governance 
and sustainability in information systems-managing the transfer and diffusion of it, 2011: Springer, pp. 155-170.

[14] R. I. Sutton and B. M. Staw, "What theory is not," Administrative science quarterly, pp. 371-384, 1995.

[15] U. Sekaran and R. Bougie, Research methods for business : a skillbuilding approach. Chichester, West Sussex, United Kingdom John Wiley \& Sons Ltd, [2016] Seventh edition., 2016.

[16] E. M. Rogers, Diffusion of innovations. New York : Free Press, c1995. 4th ed., 1995.

[17] M. G. Morris and V. Venkatesh, "Age differences in technology adoption decisions: Implications for a changing work force," (in English), Personnel Psychology, vol. 53, no. 2, pp. 375-403, Sum 2000

[18] V. Weerakkody, Z. Irani, K. Kapoor, U. Sivarajah, and Y. K. Dwivedi, "Open data and its usability: an empirical view from the Citizen's perspective," (in English), Information Systems Frontiers, vol. 19, no. 2, pp. 285-300, Apr 2017, doi: 10.1007/s10796-0169679-1.

[19] B. Gupta, G. Burns, S. G. Harris, J. D. Hershberg, and F. A. Geffen, "NOTIFICATION DISMISSAL IN AN INTERNET OF THINGS (IoT) ENVIRONMENT," ed: Google Patents, 2015.

[20] J. Schepers and M. Wetzels, "A meta-analysis of the technology acceptance model: Investigating subjective norm and moderation effects," (in English), Information \& Management, 44 (1), 90-103, Jan 2007

[21] J. C. Sipior, B. T. Ward, and R. Connolly, "The digital divide and tgovernment in the United States: using the technology acceptance model to understand usage," (in English), European Journal of Information Systems, vol. 20, no. 3, pp. 308-328, May 2011, doi: 10.1057/ejis.2010.64.

[22] S. C. Park and S. Y. Ryoo, "An empirical investigation of end-users' switching toward cloud computing: A two factor theory perspective," Computers in Human Behavior, vol. 29, no. 1, pp. 160-170, 2013.

[23] E. M. Rogers, "Diflusion ofinnovations," ed: New York: Free Press, 2003.

[24] "Yale Information Technology Services." Yale University. https://www.city.yale.edu/new-events/2019/2/13/innovation-101

[25] G. Baptista and T. Oliveira, "Understanding mobile banking: The unified theory of acceptance and use of technology combined with cultural moderators," (in English), Computers in Human Behavior, Article vol. 50, pp. 418-430, Sep 2015

[26] G. Agag and A. A. El-Masry, "Understanding consumer intention to participate in online travel community and effects on consumer intention to purchase travel online and WOM: An integration of innovation diffusion theory and TAM with trust," (in English), Computers in Human Behavior, Article vol. 60, pp. 97-111, Jul 2016

[27] S. F. Wamba, A. Anand, and L. Carter, "RFID Applications, Issues, Methods and Theory: A Review of the AIS Basket of TOP journals," Procedia Technology, Article vol. 9, pp. 421-430, 2013

[28] M. A. Hameed, S. Counsell, and S. Swift, "A conceptual model for the process of IT innovation adoption in organizations," (in English), Journal of Engineering and Technology Management, Article vol. 29, no. 3, pp. 358-390, Jul-Sep 2012

[29] K. O. L. Matthew and M. K. C. Christy, "Internet Retailing Adoption by Small-to-Medium Sized Enterprises (SMEs): A Multiple-Case Study," Information Systems Frontiers, article vol. 6, no. 4, p. 385, 2004, doi: 10.1023/B:ISFI.0000046379.58029.54.

[30] S. F. Wamba and A. T. Chatfield, "A contingency model for creating value from RFID supply chain network projects in logistics and manufacturing environments," (in English), European Journal of Information Systems, vol. 18, no. 6, pp. 615-636, Dec 2009

[31] C. Quetti, F. Pigni, and A. Clerici, "Factors affecting RFId adoption in a vertical supply chain: the case of the silk industry in Italy," (in English), Production Planning \& Control, vol. 23, no. 4, pp. 315 331, 2012, doi: 10.1080/09537287.2011.627661.

[32] Y. Liu, Y. Yang, J. N. Wei, and X. Y. Wang, "An examination on RFID innovation diffusions in Chinese public intelligent transportation services," (in English), International Journal of Mobile Communications, vol. 13, no. 5, pp. 549-566, 2015, doi: Doi 10.1504/Ijmc.2015.070971.

[33] E. D. Conrad, Willingness to use IT innovations: a hybrid approach employing diffusion of innovations and technology acceptance models. Southern Illinois University at Carbondale, 2009.

[34] B. Ryan and N. C. Gross, "The diffusion of hybrid seed corn in two Iowa communities," Rural sociology, vol. 8, no. 1, p. 15, 1943.

[35] I. Sahin, "Detailed review of Rogers' diffusion of innovations theory and educational technology-related studies based on Rogers' theory," Turkish Online Journal of Educational Technology-TOJET, vol. 5, no. 2, pp. 14-23, 2006.
[36] D. M. Berwick, "Disseminating innovations in health care," Jama, vol. 289, no. 15, pp. 1969-1975, 2003.

[37] C. Sauer and C. Lau, "Trying to adopt systems development methodologies - a case-based exploration of business users' interests," Information Systems Journal, vol. 7, no. 4, pp. 255-275, 1997.

[38] Q.-B. Sun, J. Liu, S. Li, C.-X. Fan, and J.-J. Sun, "Internet of Things: Summarize on Concepts, Architecture and Key Technology Problem [J]," Journal of Beijing University of Posts and Telecommunications, vol. 3, no. 3, pp. 1-9, 2010.

[39] J. A. Cazier, A. S. Jensen, and D. S. Dave, "The impact of consumer perceptions of information privacy and security risks on the adoption of residual RFID technologies," Communications of the Association for Information Systems, vol. 23, no. 1, p. 14, 2008.

[40] D. D. Fred, "Perceived Usefulness, Perceived Ease of Use, and User Acceptance of Information Technology," MIS Quarterly, researcharticle vol. 13, no. 3, p. 319, 1989, doi: 10.2307/249008.

[41] D. E. Gray, Doing research in the real world. Los Angeles, California: SAGE, 2014. Third edition., 2014.

[42] M. Saunders, P. Lewis, and A. Thornhill, "Research methods for business students fifth edition (fifth)," Pearson. Retrieved from https://is. cz/el/6410/leto2014/BA_BSeBM/um/Research_Methods_for_Busine ss_Students_5th_Editio $n . p d f, 2009$.

[43] R. B. Johnson, A. J. Onwuegbuzie, and L. A. Turner, "Toward a Definition of Mixed Methods Research," (in English), Journal of Mixed Methods Research, vol. 1, no. 2, pp. 112-133, Apr 2007,

[44] M. E. Smith, R. Thorpe, and P. Jackson, "Management research," LA: SAGE, 2008.

[45] A. Tashakkori and C. Teddlie, "Integrating qualitative and quantitative approaches to research," The SAGE handbook of applied social research methods, vol. 2, pp. 283-317, 2009.

[46] J. W. Creswell and V. L. P. Clark, Designing and conducting mixed methods research. Sage publications, 2017.

[47] F. Tuli, "The basis of distinction between qualitative and quantitative research in social science: Reflection on ontological, epistemological and methodological perspectives," Ethiopian Journal of Education and Sciences, vol. 6, no. 1, 2010.

[48] D. Straub, M.-C. Boudreau, and D. Gefen, "Validation guidelines for IS positivist research," Communications of the Association for Information systems, vol. 13, no. 1, p. 24, 2004.

[49] S. W. Vanderstoep and D. D. Johnson, Research methods for everyday life: Blending qualitative and quantitative approaches. John Wiley \& Sons, 2008.

[50] J. Hair Jr, W. Black, B. Babin, and R. Anderson, "Multivariate data analysis a global perspective. Pearson Education Inc," USR, New Jersey, vol. 7458, 2010.

[51] R. K. Yin, Case study research : design and methods. Los Angeles : SAGE, [2014] Fifth edition., 2014.

[52] M. Saunders, P. Lewis, and A. Thornhill, Research methods for business students. Harlow : Financial Times Prentice Hall, 2012.7th ed., 2016.

[53] C. Cassell and G. Symon, "Essential Guide to Qualitative Methods in Organizational Research," ed: SAGE Publications Ltd, 2004.

[54] S. J. Miah, J. G. Gammack, and J. McKay, "A Metadesign theory for Tailorable decision support," Journal of the Association for Information Systems, vol. 20, no. 5, p. 4, 2019.

[55] S.J. Miah, J. Shen, JW Lamp, D. Kerr, and J.G. Gammack, "Emerging Insights of Health Informatics Research: A Literature Analysis for Outlining New Themes", Australasian Journal of Information Systems 23, 1-18, 2019.

[56] S. J. Miah, "A Demand-Driven Cloud-Based Business Intelligence for Healthcare Decision Making", Hand-book of Research on DemandDriven Web Services: Theory, Technologies, and Applications: Theory, Technologies, and Applications, 324, 2014.

[57] MR Islam, S.J. Miah, ARM. Kamal, and O. Burmeister, "A Design Construct for Developing Approaches to Measure Mental Health Conditions", Australasian Journal of Information Systems, 23, 1-22, 2019. 